\title{
The Commutation Matrices of Elements in Kronecker Quaternion Group
}

\author{
Yanita Yanita ${ }^{*}$, Eka Purwanti $^{2}$, Lyra Yulianti $^{3}$ \\ 1,2,3 Department of Mathematics, Faculty of Mathematic and Natural Science, Andalas University, \\ Kampus Unand Limau Manis, Padang 25163, Indonesia \\ ${ }^{*}$ Corresponding author. Email: yanita@sci.unand.ac.id
}

\begin{abstract}
This article discusses the commutation matrix in the Kronecker quaternion group; that is, a non-abelian group whose 32 elements are $4 \times 4$ matrices, with entries in the set of complex numbers. This paper aims to describe the commutation matrices obtained concerning the matrices in this group. The commutation matrix is a permutation matrix that associates the relationship between the vec of matrix and vec of its transpose. Based on the classification of matrices in the Kronecker quaternion group, there are 16 classifications of commutation matrices for the matrices in this group.

Keywords:

Kronecker Quaternion Group; Permutation Matrix; Commutation Matrix; Vec Matrix

How to Cite:

Y. Yanita, E. Purwanti, and L. Yulianti, "The Commutation Matrices of Elements in Kronecker Quaternion Group", Jambura J. Math., vol. 4, No. 1, pp. 135-144, 2022, doi: https://doi.org/10.34312/jjom.v4i1.12004
\end{abstract}

\section{Introduction}

The representation of quaternion group is a group quaternion written in another form (see $[1,2])$. A new group can be created using the Kronecker product for each of the two elements from this group. The new group is called the Kronecker quaternion group. Several studies can be carried out from this group, namely based on the characteristics that appear in the group and also based on the elements of the group.

The Kronecker quaternion group was introduced by Yanita, et.al [3]. This group is a non-abelian consisting of $324 \times 4$ matrices. The study of this group was continued by Yanita [4], which is related to writing this group in the form of a generator and relator. Furthermore, Adrianda [5] and Zakiya [6] review this group based on directed graphs and the second homotopy module, namely group studies presented geometrically with graph and picture forms. These studies have not linked the Kronecker quaternion group based on the existing matrices in the group, but rather to other forms of groups that are presented differently.

Furthermore, inspired by Wang and Davis [7], we started the study by paying attention to the elements in this Kronecker quaternion group, namely by compiling a new matrix, the partition matrix, with the sub-matrices being the matrices in this group. The result

e-ISSN: 2656-1344 (C) 2022 Y. Yanita, E. Purwanti, L. Yulianti | Under the license CC BY-NC 4.0

Received: 12 November 2021 | Accepted: 20 December 2021 | Online: 1 January 2022 
is that the two $8 \times 8$ partition matrices $A$ and $B$, whose submatrices are matrices in the Kronecker quaternion group, form $A^{T} A=B B^{T}$. This shape is also generated when the locations of the varying permutation matrices are coupled to the submatrix, so that the general result of this study is a specific form, $A^{T} A=B B^{T}$, even though the submatrix is not a symmetric matrix.

In the previous study, the results were obtained by arranging the matrices in the Kronecker quaternion group into a new matrix (see [8]). This paper then tries to develop a study while still considering the matrices in the Kronecker quaternion group to determine the commutation matrix for each matrix in the group. Since the form of the matrix in this group can be classified based on the symmetry and skew-symmetry properties, the result is the form of the commutation matrix generated based on this classification. Besides that, the diagonal form of the matrix (main and secondary) also forms the basis for the formation of this commutation matrix.

The organizing of this paper as follows: In Section 2, it is introduced a lot of basic concepts and notations of vec matrix, permutation matrix and commutation matrix, which will be used in Section 3. In Section 3, it is presented the commutation matrix for each matrix in the Kronecker quaternion group by first classifying these matrices.

\section{Methods}

The research methods are based on the study of literature, which is related to the transformation of the permutation matrix on the vec matrix. The first step of this research is to determine the shape/pattern of the commutation matrix for the symmetry and skew-symmetry. Since the symmetry matrix in the Kronecker quaternion group is in the form of a diagonal matrix (main and secondary), the commutation matrix pattern is classified into these two forms.

The second step of this research is to apply the pattern obtained in the first step to all the elements (matrices) in the Kronecker quaternion group.

In this Section, we present some definitions, properties and theorems used to obtain the result.

Definition 1. [9] Let $A=\left[a_{i j}\right]$ be an $m \times n$ matrix, and $A_{j}$ the column of $A$. The $\operatorname{vec}(A)$ is the $n$ column vector, i.e

$$
\operatorname{vec}(A)=\left[\begin{array}{c}
A_{1} \\
A_{2} \\
\vdots \\
A_{n}
\end{array}\right]
$$

Let $S_{n}$ denote the set of all permutation of the $n$ element set $[n]:=\{1,2, \ldots, n\}$. A permutation is one-to-one function from $[n]$ onto $[n]$. Permutation of finite sets are usually given by listing of each element of the domain and its corresponding functional value. For example, we define a permutation $\sigma$ of the set $[n]:=\{1,2,3,4,5,6\}$ by specifying $\sigma(1)=5, \sigma(2)=3, \sigma(3)=1, \sigma(4)=6, \sigma(5)=2, \sigma(6)=4$. A more convenient way to express this correspondence is towrite $\sigma$ in array form as

$$
\sigma=\left[\begin{array}{llllll}
1 & 2 & 3 & 4 & 5 & 6 \\
5 & 3 & 1 & 6 & 2 & 4
\end{array}\right]
$$


There is another notation commonly used to specify permutation. It is called cycle notation. Cycle notation has theoretical advantages in that certain important properties of the permutation can be readily determined when cycle notation is used. For example, permutation in (1) can be written as $\sigma=\left(\begin{array}{llll}1 & 5 & 2 & 3\end{array}\right)\left(\begin{array}{ll}4 & 6\end{array}\right)$. For detail see [10].

Theorem 1. [10] Let $\pi$ and $\sigma$ be a permutation in $S_{n}$, then $P(\pi) P(\sigma)=P(\pi \sigma)$.

If $\sigma$ is a permutation, we have the identity matrix as follows:

Definition 2. [11] Let $\sigma$ be a permutation in $S_{n}$. Define the permutation matrix $P(\sigma)=$ $\left[\delta_{i, \sigma(j)}\right], \delta_{i, \sigma(j)}=$ entry $_{i, j}(P(\sigma))$ where

$$
\delta_{i, \sigma(j)}= \begin{cases}1 & \text { if } i=\sigma(j) \\ 0 & \text { if } i \neq \sigma(j)\end{cases}
$$

Example 1. Let $n:=\{1,2,3\}$ and $\sigma=\left(\begin{array}{lll}1 & 2 & 3\end{array}\right)$.

$P(123)=\left[\delta_{i, \sigma(j)}\right]$ and $\delta_{i, \sigma(j)}=\left\{\begin{array}{l}1 \text { if } i=\sigma(j) \\ 0 \text { if } i \neq \sigma(j)\end{array}\right.$

( 1 to $2 ; 2$ to $3 ; 3$ to $1 ; \sigma(1)=2, \sigma(2)=3, \sigma(3)=1$ )

$$
\begin{gathered}
\text { ent }_{11}(P(\sigma))=\delta_{1, \sigma(1)}=0(\sigma(1)=2) ; \text { ent }_{12}(P(\sigma))=\delta_{1, \sigma(2)}=0(\sigma(2)=3) ; \\
\text { ent }_{13}(P(\sigma))=\delta_{1, \sigma(3)}=1(\sigma(3)=1) \text { ent }_{21}(P(\sigma))=\delta_{2, \sigma(1)}=1(\sigma(1)=2) ; \\
\text { ent }_{22}(P(\sigma))=\delta_{2, \sigma(2)}=0(\sigma(2)=3) \text { int }_{23}(P(\sigma))=\delta_{2, \sigma(3)}=0(\sigma(3)=1) ; \\
\text { ent }_{31}(P(\sigma))=\delta_{3, \sigma(1)}=0(\sigma(1)=2) ; \text { ent }_{32}(P(\sigma))=\delta_{3, \sigma(2)}=0(\sigma(2)=3) ; \\
\text { ent }_{33}(P(\sigma))=\delta_{3, \sigma(3)}=0(\sigma(3)=1) ;
\end{gathered}
$$

So we have $P(123)=\left[\begin{array}{lll}\delta_{12} & \delta_{13} & \delta_{11} \\ \delta_{22} & \delta_{23} & \delta_{21} \\ \delta_{32} & \delta_{33} & \delta_{31}\end{array}\right]=\left[\begin{array}{lll}0 & 0 & 1 \\ 1 & 0 & 0 \\ 0 & 1 & 0\end{array}\right]$

The commutation matrix is a kind of permutation matrix of order $m n$ expressed as a block matrix where each block is of the same size and has a unique 1 in it.

Definition 3. [12] A permutation matrix $P$ is called a commutation matrix of matrix, $m \times n$, if it satisfies the following condition:

1. $P=\left[A_{i j}\right]$ is an $m \times n$ block matrix with each block $A_{i j}$ be a $n \times m$ matrix.

2. For each $i \in[m], j \in[n], A_{i j}=\left(a_{s, t}(i, j)\right)$ is a $(0,1)$ matrix with a unique 1 which lies at the position $(j, i)$.

We denote this commutation matrix by $K_{m, n}$ and thus a communication matrix is of size $m n \times m n$.

Example 2. Matrix $K_{3,2}$ is a $6 \times 6$ permutation matrix partitioned by a $3 \times 2$ block matrix, i.e:

$$
K_{3,2}=\left[\begin{array}{ll}
A_{11} & A_{12} \\
A_{21} & A_{22} \\
A_{31} & A_{32}
\end{array}\right]
$$


The Commutation Matrices of Elements in Kronecker Quaternion Group...

where $A_{i j}=\left(a_{s, t}{ }^{(i, j)}\right)$ is a $2 \times 3$ matrix whose unique non zero entry is $a_{j, i}{ }^{(i, j)}=1$. Specipically

$$
K_{3,2}=\left[\begin{array}{llllll}
1 & 0 & 0 & 0 & 0 & 0 \\
0 & 0 & 0 & 1 & 0 & 0 \\
0 & 1 & 0 & 0 & 0 & 0 \\
0 & 0 & 0 & 0 & 1 & 0 \\
0 & 0 & 1 & 0 & 0 & 0 \\
0 & 0 & 0 & 0 & 0 & 1
\end{array}\right]
$$

The definition of the commutation matrix is given in different way by Zhang [13], that is

$$
K_{m, n}=\left[\begin{array}{c}
I_{m} \otimes \boldsymbol{e}_{1 n^{T}} \\
I_{m} \otimes \boldsymbol{e}_{2 n}^{T} \\
\vdots \\
I_{m} \otimes \boldsymbol{e}_{m n}^{T}
\end{array}\right]
$$

where $I_{m}$ is an identity matrix and $\boldsymbol{e}_{i n}$ is an $n$-dimensional column vector which has 1 in the $i^{\text {th }}$ position and $0^{\prime} \mathrm{s}$ elsewhere; that is

$$
\boldsymbol{e}_{\text {in }}=[0,0, \ldots, 0,1,0, \ldots, 0]^{T}
$$

and

$$
I_{m} \otimes \boldsymbol{e}_{i n}{ }^{T}=\left[a_{i j} \boldsymbol{e}_{i n}^{T}\right], a_{i j} \in I_{m} .
$$

Lemma 1. [14] Let $K_{m, n}$ be a commutation matrix. Then

1. $K_{m, n}{ }^{T}=K_{n, m}$ and $K_{m, n} K_{n, m}=I_{m n}$

2. $K_{m, 1}=K_{1, m}=I_{m}$

The following theorem present a linear relationship between $\operatorname{vec}(A)$ and $\operatorname{vec}\left(A^{T}\right)$ through the commutation matrix $K_{m, n}$.

Theorem 2. [15] Let $m, n \in \mathbb{Z}^{+}$and $A$ be a $m \times n$ matrix, then $K_{m n}$ vec $(A)=\operatorname{vec}\left(A^{T}\right)$.

The second step of this research is to apply the pattern obtained in the first step to all the elements (matrices) in the Kronecker quaternion group. Next, the final step is to determine the type of commutation matrix for each matrix in the Kronecker Quaternion Group.

\section{Results and Discussion}

We present the Kronecker quartenion group, i.e.

$\mathrm{G}=\left\{A_{k}=\left[a_{i j}\right] \mid i, j=1,2,3,4, k=1,2, \ldots, 32\right\}$ where $A_{k}$ as follows:

$$
A_{1}=\left[\begin{array}{llll}
1 & 0 & 0 & 0 \\
0 & 1 & 0 & 0 \\
0 & 0 & 1 & 0 \\
0 & 0 & 0 & 1
\end{array}\right], A_{2}=\left[\begin{array}{cccc}
-1 & 0 & 0 & 0 \\
0 & -1 & 0 & 0 \\
0 & 0 & -1 & 0 \\
0 & 0 & 0 & -1
\end{array}\right]
$$




$$
\begin{aligned}
& A_{3}=\left[\begin{array}{cccc}
i & 0 & 0 & 0 \\
0 & -i & 0 & 0 \\
0 & 0 & i & 0 \\
0 & 0 & 0 & -i
\end{array}\right], A_{4}=\left[\begin{array}{cccc}
-i & 0 & 0 & 0 \\
0 & i & 0 & 0 \\
0 & 0 & -i & 0 \\
0 & 0 & 0 & i
\end{array}\right] \\
& A_{5}=\left[\begin{array}{cccc}
0 & 1 & 0 & 0 \\
-1 & 0 & 0 & 0 \\
0 & 0 & 0 & 1 \\
0 & 0 & -1 & 0
\end{array}\right], A_{6}=\left[\begin{array}{cccc}
0 & -1 & 0 & 0 \\
1 & 0 & 0 & 0 \\
0 & 0 & 0 & -1 \\
0 & 0 & 1 & 0
\end{array}\right] \text {, } \\
& A_{7}=\left[\begin{array}{cccc}
0 & i & 0 & 0 \\
i & 0 & 0 & 0 \\
0 & 0 & 0 & i \\
0 & 0 & i & 0
\end{array}\right], A_{8}=\left[\begin{array}{cccc}
0 & -i & 0 & 0 \\
-i & 0 & 0 & 0 \\
0 & 0 & 0 & -i \\
0 & 0 & -i & 0
\end{array}\right] \text {, } \\
& A_{9}=\left[\begin{array}{cccc}
i & 0 & 0 & 0 \\
0 & i & 0 & 0 \\
0 & 0 & -i & 0 \\
0 & 0 & 0 & -i
\end{array}\right], A_{10}=\left[\begin{array}{cccc}
-i & 0 & 0 & 0 \\
0 & -i & 0 & 0 \\
0 & 0 & i & 0 \\
0 & 0 & 0 & i
\end{array}\right] \text {, } \\
& A_{11}=\left[\begin{array}{cccc}
-1 & 0 & 0 & 0 \\
0 & 1 & 0 & 0 \\
0 & 0 & 1 & 0 \\
0 & 0 & 0 & -1
\end{array}\right], A_{12}=\left[\begin{array}{cccc}
1 & 0 & 1 & 0 \\
0 & -1 & 0 & 0 \\
0 & 0 & -1 & 0 \\
0 & 0 & 0 & 1
\end{array}\right] \text {, } \\
& A_{13}=\left[\begin{array}{cccc}
0 & i & 0 & 0 \\
-i & 0 & 0 & 0 \\
0 & 0 & 0 & -i \\
0 & 0 & i & 0
\end{array}\right], A_{14}=\left[\begin{array}{cccc}
0 & -i & 0 & 0 \\
i & 0 & 0 & 0 \\
0 & 0 & 0 & i \\
0 & 0 & -i & 0
\end{array}\right] \text {, } \\
& A_{15}=\left[\begin{array}{cccc}
0 & -1 & 0 & 0 \\
-1 & 0 & 0 & 0 \\
0 & 0 & 0 & 1 \\
0 & 0 & 1 & 0
\end{array}\right], A_{16}=\left[\begin{array}{cccc}
0 & 1 & 0 & 0 \\
1 & 0 & 0 & 0 \\
0 & 0 & 0 & -1 \\
0 & 0 & -1 & 0
\end{array}\right] \text {, } \\
& A_{17}=\left[\begin{array}{cccc}
0 & 0 & 1 & 0 \\
0 & 0 & 0 & 1 \\
-1 & 0 & 0 & 0 \\
0 & -1 & 0 & 0
\end{array}\right], A_{18}=\left[\begin{array}{cccc}
0 & 0 & -1 & 0 \\
0 & 0 & 0 & -1 \\
1 & 0 & 0 & 0 \\
0 & 1 & 0 & 0
\end{array}\right] \text {, } \\
& A_{19}=\left[\begin{array}{cccc}
0 & 0 & i & 0 \\
0 & 0 & 0 & -i \\
-i & 0 & 0 & 0 \\
0 & i & 0 & 0
\end{array}\right], A_{20}=\left[\begin{array}{cccc}
0 & 0 & -i & 0 \\
0 & 0 & 0 & i \\
i & 0 & 0 & 0 \\
0 & -i & 0 & 0
\end{array}\right] \text {, } \\
& A_{21}=\left[\begin{array}{cccc}
0 & 0 & 0 & 1 \\
0 & 0 & -1 & 0 \\
0 & -1 & 0 & 0 \\
1 & 0 & 0 & 0
\end{array}\right], A_{22}=\left[\begin{array}{cccc}
0 & 0 & 0 & -1 \\
0 & 0 & 1 & 0 \\
0 & 1 & 0 & 0 \\
-1 & 0 & 0 & 0
\end{array}\right] \text {, }
\end{aligned}
$$


The Commutation Matrices of Elements in Kronecker Quaternion Group...

$$
\begin{aligned}
& A_{23}=\left[\begin{array}{cccc}
0 & 0 & 0 & i \\
0 & 0 & i & 0 \\
0 & -i & 0 & 0 \\
-i & 0 & 0 & 0
\end{array}\right], A_{24}=\left[\begin{array}{cccc}
0 & 0 & 0 & -i \\
0 & 0 & -i & 0 \\
0 & i & 0 & 0 \\
i & 0 & 0 & 0
\end{array}\right] \\
& A_{25}=\left[\begin{array}{cccc}
0 & 0 & i & 0 \\
0 & 0 & 0 & i \\
i & 0 & 0 & 0 \\
0 & i & 0 & 0
\end{array}\right], A_{26}=\left[\begin{array}{cccc}
0 & 0 & -i & 0 \\
0 & 0 & 0 & -i \\
-i & 0 & 0 & 0 \\
0 & -i & 0 & 0
\end{array}\right] \text {, } \\
& A_{27}=\left[\begin{array}{cccc}
0 & 0 & -1 & 0 \\
0 & 0 & 0 & 1 \\
-1 & 0 & 0 & 0 \\
0 & 1 & 0 & 0
\end{array}\right], A_{28}=\left[\begin{array}{cccc}
0 & 0 & 1 & 0 \\
0 & 0 & 0 & -1 \\
1 & 0 & 0 & 0 \\
0 & -1 & 0 & 0
\end{array}\right] \\
& A_{29}=\left[\begin{array}{cccc}
0 & 0 & 0 & i \\
0 & 0 & -i & 0 \\
0 & i & 0 & 0 \\
-i & 0 & 0 & 0
\end{array}\right], A_{30}=\left[\begin{array}{cccc}
0 & 0 & 0 & -i \\
0 & 0 & i & 0 \\
0 & -i & 0 & 0 \\
i & 0 & 0 & 0
\end{array}\right] \text {, } \\
& A_{31}=\left[\begin{array}{cccc}
0 & 0 & 0 & -1 \\
0 & 0 & -1 & 0 \\
0 & -1 & 0 & 0 \\
-1 & 0 & 0 & 0
\end{array}\right], A_{32}=\left[\begin{array}{llll}
0 & 0 & 0 & 1 \\
0 & 0 & 1 & 0 \\
0 & 1 & 0 & 0 \\
1 & 0 & 0 & 0
\end{array}\right] \text {. }
\end{aligned}
$$

The classification of the elements in $\mathrm{G}$ are:

1. Symmetric matrix (main diagonal): $A_{1}, A_{2}, A_{3}, A_{4}, A_{9}, A_{10}, A_{11}, A_{12}, A_{21}, A_{22}$

2. Symmetric matrix (secondary diagonal): $A_{7}, A_{8}, A_{15}, A_{16}, A_{25}, A_{26}, A_{27}, A_{28}, A_{31}$, $A_{32}$.

3. Skew-Symmetrix matrix (secondary diagonal): $A_{5}, A_{6}, A_{13}, A_{14}, A_{17}, A_{18}, A_{19}, A_{20}$, $A_{23}, A_{24}, A_{29}, A_{30}$.

Based on the classification above, the elements in the Kronecker quaternion group are also divided based on the same elements in certain entries, i.e.:

1. Symmetri matrix (main diagonal), where

$$
\begin{aligned}
& \text { - } a_{11}=a_{22}=a_{33}=a_{44}: A_{1}, A_{2} . \\
& \text { - } a_{11}=a_{33} \text { and } a_{22}=a_{44}: A_{3}, A_{4} . \\
& \text { - } a_{11}=a_{22} \text { and } a_{33}=a_{44}: A_{9}, A_{10} . \\
& \text { - } a_{11}=a_{44} \text { and } a_{22}=a_{33}: A_{11}, A_{12} \text {. }
\end{aligned}
$$

2. Symmetri matrix (secondary diagonal), where

- $a_{21}=a_{12}=a_{43}=a_{34}: A_{7}, A_{8}$.

- $a_{41}=a_{32}=a_{23}=a_{14}: A_{31}, A_{32}$.

- $a_{31}=a_{13}=a_{42}=a_{24}: A_{25}, A_{26}$.

- $a_{21}=a_{12}$ and $a_{43}=a_{34}: A_{15}, A_{16}$.

- $a_{41}=a_{14}$ and $a_{32}=a_{23}: A_{21}, A_{22}$.

- $a_{31}=a_{13}$ and $a_{42}=a_{24}: A_{27}, A_{28}$.

3. Skew-symmetric matrix (secondary diagonal), where

- $a_{21}=a_{34}$ and $a_{12}=a_{43}: A_{13}, A_{14}$.

- $a_{31}=a_{42}$ and $a_{13}=a_{24}: A_{17}, A_{18}$.

- $a_{24}=a_{31}$ and $a_{13}=a_{42}: A_{19}, A_{20}$. 
- $a_{41}=a_{32}$ and $a_{23}=a_{14}: A_{23}, A_{24}$.

- $a_{41}=a_{23}$ and $a_{32}=a_{14}: A_{29}, A_{30}$.

- $a_{21}=a_{43}$ and $a_{12}=a_{34}: A_{5}, A_{6}$.

We have three theorems to determine the commutation matrix of matrices in the Kronecker quaernion group.

Theorem 3. Let $A$ be a $n \times n$ matrix. Then the product of permutation matrices $P(((j-1) n+i)((i-1) n+j))$ is a commutation matrix $K_{n, n}$ of $A$.

Proof. Consider that, the $i j^{\text {th }}$ element of $A$ is the $((j-1) n+i)^{\text {th }}$ element of $\operatorname{vec}(A)$ and the $i j^{\text {th }}$ element of $A^{T}$ is the $((i-1) n+j)^{t h}$ element of $\operatorname{vec}(A)$. Thus, $K_{n, n}$ is the permutation matrix that takes elements $((j-1) n+i)^{\text {th }}$ to $((i-1) n+j)^{t h}$ where $i, j=1,2, \ldots, n$.

The proof divided into two, i.e for $i=j$ and $i \neq j$.

- For $i=j$, we have the permutation matrix that takes elements $((i-1) n+i)^{\text {th }}$ to $((i-1) n+i)^{t h}$. So, the permutation matrix takes 1 to $1, n+2$ to $n+2, \ldots, n^{2}$ to $n^{2}$. Then, we have the permutation matrix $P(((i-1) n+i)((i-1) n+i))=I_{n^{2}}=$ $K_{n, n}$

- For $i \neq j$, Let $i=r, j=s ; r \neq s ; r, s=1,2, \ldots, n$, then we have permutation matrix that takes elements $((s-1) n+r)^{\text {th }}$ to $((r-1) n+s)^{\text {th }}$.

Let $i=s, j=r ; r \neq s ; r, s=1,2, \ldots, n$, then we have permutation matrix that takes elements $((r-1) n+s)^{t h}$ to $((s-1) n+r)^{t h}$.

$\mathrm{t}$ Base on these, we have the permutation matrix takes elements $((s-1) n+r)^{\text {th }}$ to $((r-1) n+s)^{t h}$ and $((r-1) n+s)^{t h}$ to $((s-1) n+r)^{t h}$. In other words, we have $P(((s-1) n+r)((r-1) n+s))$, or in generally $P(((j-1) n+i)((i-1) n+j))$ for $i>j$. Thus,

$$
\begin{gathered}
K_{n, n}=P_{i=j}(((j-1) n+i)((i-1) n+j)) P_{i>j}(((j-1) n+i) \quad((i-1) n+j)) \\
=P_{i \geq j}(((j-1) n+i)((i-1) n+j))
\end{gathered}
$$

Theorem 4. Let $A$ be a matrix $m \times n$ such that $a_{r}$ is the $r^{\text {th }}$ entry in $\operatorname{vec}(A)$ and $a_{s}$ is the $s^{\text {th }}$ entry in vec $\left(A^{T}\right)$ where $a_{r}=a_{s}$. If the commutation matrix $K_{m n}$ canges the location of $a_{r}$ in $\operatorname{vec}(A)$ to the $a_{s}$ entry in $\operatorname{vec}\left(A^{T}\right)$ then the $a_{s r}$ entry in $K_{m n}$ is 1.

Proof. The elements of the commutation matrix are 0 and 1 . The $a_{i j}$ element in $A$ are $((j-1) m+i)^{t h}$ element in in the row in vec $(A)$ and the $((i-1) n+j)^{t h}$ element in row in $\operatorname{vec}\left(A^{T}\right)$. Let $r=(j-1) m+i$ and $s=(i-1) n+j$ so that $a_{r}=a_{s}$. Based on matrix multiplication, the element $a_{s r}$ in $K_{m n}$ is 1 which acts to make the element $a_{r}$ in vec $(A)$ become the element $a_{S}$ in vec $\left(A^{T}\right)$.

The illustration for Theorem 4

Let $A=\left[\begin{array}{cccc}1 & 0 & 0 & 0 \\ 0 & 1 & 0 & 0 \\ 0 & 0 & 1 & 0 \\ 0 & 0 & 0 & 1\end{array}\right]$. 
The Commutation Matrices of Elements in Kronecker Quaternion Group...

We have vec $(A)=\left[\begin{array}{l}1 \\ 0 \\ 0 \\ 0 \\ 0 \\ 1 \\ 0 \\ 0 \\ 0 \\ 0 \\ 1 \\ 0 \\ 0 \\ 0 \\ 0 \\ 1\end{array}\right]=\left[\begin{array}{l}a_{1} \\ a_{2} \\ a_{3} \\ a_{4} \\ a_{5} \\ a_{6} \\ a_{7} \\ a_{8} \\ a_{9} \\ a_{10} \\ a_{11} \\ a_{12} \\ a_{13} \\ a_{14} \\ a_{15} \\ a_{16}\end{array}\right]$ and vec $\left(A^{T}\right)=\left[\begin{array}{l}1 \\ 0 \\ 0 \\ 0 \\ 0 \\ 1 \\ 0 \\ 0 \\ 0 \\ 0 \\ 1 \\ 0 \\ 0 \\ 0 \\ 0 \\ 1\end{array}\right]=\left[\begin{array}{l}a_{1} \\ a_{2} \\ a_{3} \\ a_{4} \\ a_{5} \\ a_{6} \\ a_{7} \\ a_{8} \\ a_{9} \\ a_{10} \\ a_{11} \\ a_{12} \\ a_{13} \\ a_{14} \\ a_{15} \\ a_{16}\end{array}\right]$.

Based on Theorem 3, we have $K_{4,4}=P(25)(39)(413)(7$ 10)(8 14)(12 15). Thus,

$$
K_{4,4} \operatorname{vec}(A)=\left[\begin{array}{llllllllllllllll}
1 & 0 & 0 & 0 & 0 & 0 & 0 & 0 & 0 & 0 & 0 & 0 & 0 & 0 & 0 & 0 \\
0 & 0 & 0 & 0 & 1 & 0 & 0 & 0 & 0 & 0 & 0 & 0 & 0 & 0 & 0 & 0 \\
0 & 0 & 0 & 0 & 0 & 0 & 0 & 0 & 1 & 0 & 0 & 0 & 0 & 0 & 0 & 0 \\
0 & 0 & 0 & 0 & 0 & 0 & 0 & 0 & 0 & 0 & 0 & 0 & 1 & 0 & 0 & 0 \\
0 & 1 & 0 & 0 & 0 & 0 & 0 & 0 & 0 & 0 & 0 & 0 & 0 & 0 & 0 & 0 \\
0 & 0 & 0 & 0 & 0 & 1 & 0 & 0 & 0 & 0 & 0 & 0 & 0 & 0 & 0 & 0 \\
0 & 0 & 0 & 0 & 0 & 0 & 0 & 0 & 0 & 1 & 0 & 0 & 0 & 0 & 0 & 0 \\
0 & 0 & 0 & 0 & 0 & 0 & 0 & 0 & 0 & 0 & 0 & 0 & 0 & 1 & 0 & 0 \\
0 & 0 & 1 & 0 & 0 & 0 & 0 & 0 & 0 & 0 & 0 & 0 & 0 & 0 & 0 & 0 \\
0 & 0 & 0 & 0 & 0 & 0 & 1 & 0 & 0 & 0 & 0 & 0 & 0 & 0 & 0 & 0 \\
0 & 0 & 0 & 0 & 0 & 0 & 0 & 0 & 0 & 0 & 1 & 0 & 0 & 0 & 0 & 0 \\
0 & 0 & 0 & 0 & 0 & 0 & 0 & 0 & 0 & 0 & 0 & 0 & 0 & 0 & 1 & 0 \\
0 & 0 & 0 & 1 & 0 & 0 & 0 & 0 & 0 & 0 & 0 & 0 & 0 & 0 & 0 & 0 \\
0 & 0 & 0 & 0 & 0 & 0 & 0 & 1 & 0 & 0 & 0 & 0 & 0 & 0 & 0 & 0 \\
0 & 0 & 0 & 0 & 0 & 0 & 0 & 0 & 0 & 0 & 0 & 1 & 0 & 0 & 0 & 0 \\
0 & 0 & 0 & 0 & 0 & 0 & 0 & 0 & 0 & 0 & 0 & 0 & 0 & 0 & 0 & 1
\end{array}\right]\left[\begin{array}{l}
1 \\
0 \\
0 \\
0 \\
0 \\
1 \\
0 \\
0 \\
0 \\
0 \\
1 \\
0 \\
0 \\
0 \\
0 \\
0 \\
0 \\
0 \\
1 \\
0 \\
0 \\
0 \\
0 \\
1 \\
0 \\
0 \\
0 \\
0 \\
1
\end{array}\right]=\operatorname{vec}\left(A^{T}\right)
$$

It is known that $K_{4,4}$ places the $2^{\text {nd }}$ element in $\operatorname{vec}(A)$ into the $5^{\text {th }}$ element in $\operatorname{vec}\left(A^{T}\right)$ so that the $5^{\text {th }}$ row and the $2^{\text {nd }}$ column of the $K_{4,4}$ is 1 . By considering the same entries in the matrix, we can find several ways of placing the entries in $\operatorname{vec}(A)$ to $\operatorname{vec}\left(A^{T}\right)$, so that the commutation matrix is not unique.

Theorem 5. Let $A$ be any matrix of size $m \times n$ with $k$ different elements, i.e $a_{1}, a_{2}, \ldots, a_{k}$, $k \leq m n$. If $\left|a_{t}\right|=s_{t}$ where $t=1,2, \ldots, k$, then the number of possible commutation matrices of $A$ is $s_{1} ! s_{2} ! \ldots s_{k} !$

Proof. Let $a_{i j}=a_{1}=a_{2} \cdots=a_{t}$ where $t=1,2, \ldots, k$ and $\left|a_{t}\right|=s_{t}$. Based on Theorem 3 and Theorem 4, commutation matrix $K_{n, n}$ places the $((j-1) m+i)^{\text {th }}$ element in vec $(A)$ into the $((i-1) n+j)^{t h}$ in vec $\left(A^{T}\right), i=1,2, \ldots, m$ and $j=1,2, \ldots, n$. There are $s !$ way to put $s_{t}$ element from $\operatorname{vec}(A)$ to $\operatorname{vec}\left(A^{T}\right)$, that is there are $s$ ! permutation matrices (read commutation matrices) for placing the same element, namely $a_{t}$ such that it transforms $\operatorname{vec}(A)$ into $\operatorname{vec}\left(A^{T}\right)$. Therefore, the different permutation matrices that transform 


\section{Y. Yanita, et.al}

$\operatorname{vec}(A)$ to $\operatorname{vec}\left(A^{T}\right)$ are $s_{1} ! s_{2} ! \ldots s_{k} !$

Base on Theorem 3, Theorem 4 and Theorem 5, we have the commutation matrix for elements of Kronecker quaternion group, as follows:

Symmetry matrix (main diagonal)

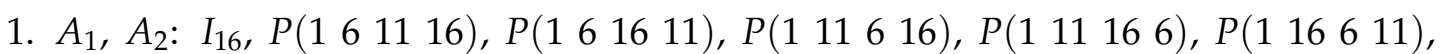
$P\left(\begin{array}{llll}1 & 16 & 11 & 6\end{array}\right), P\left(\begin{array}{lll}1 & 11 & 6\end{array}\right), P\left(\begin{array}{lll}1 & 16 & 6\end{array}\right), P\left(\begin{array}{lll}1 & 16 & 11\end{array}\right), P\left(\begin{array}{lll}1 & 6 & 16\end{array}\right), P\left(\begin{array}{lll}1 & 16 & 11\end{array}\right)$, $P(111$ 16),$P(616$ 11) $P(611$ 16) $P(16), P(11$ 16), $P(1$ 11) $P(116), P(6$ 11), $P(616), P(16)(11$ 16) $, P(111)(616), P(116)(611)$

2. $A_{3}, A_{4}: I_{16}, P(111), P(6 \quad 16), P\left(\begin{array}{ll}1 & 11\end{array}\right)\left(\begin{array}{ll}6 & 16\end{array}\right)$

3. $A_{9}, A_{10}: I_{16}, P\left(\begin{array}{ll}1 & 6\end{array}\right), P\left(11\right.$ 16),$P\left(\begin{array}{ll}1 & 6\end{array}\right)\left(\begin{array}{ll}11 & 16\end{array}\right)$.

4. $A_{11}, A_{12}: I_{16}, P(116), P(6 \quad 11), P\left(\begin{array}{ll}1 & 16\end{array}\right)\left(\begin{array}{ll}6 & 11\end{array}\right)$

Symmetry matrix (secondary diagonal)

1. $A_{7}, A_{8}: I_{16}, P(2512$ 15), $P(2515$ 12), $P(2125$ 15), $P(21215$ 5), $P(2155$ 12), $P(21512$ 5), $P(2125), P(215$ 5),$P(215$ 12) $P(25$ 15), $P(215$ 12) $P(21215)$, $P(515$ 12),$P(512$ 15) $P(2$ 5), $P(12$ 15) $P(2$ 12) $P(2$ 15),$P(5$ 12) $P(5$ 15), $P(25)(12$ 15) $, P(212)(515), P(215)(512)$.

2. $A_{31}, A_{32}: I_{16}, P(471013), P(4713$ 10), , $P(410713), P(410137), P(413710)$, $P(413107, P(4107), P(4137), P(413$ 10), $P(47$ 13), $P(413$ 10), $P(41013)$, $P(713$ 10), $P(71013), P(47), P(1013), P(410), P(413), P(710), P(713)$, $P(47)(1013), P(410)(713), P(413)(710)$.

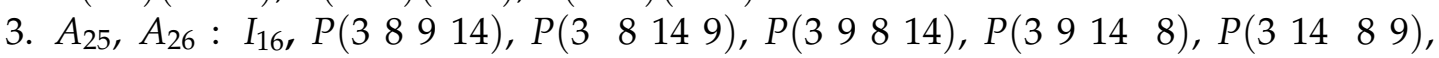
$P(3149$ 8), $P(398), P(314$ 8) $, P(3149), P(3814), P(3149), P(3914), P(8149)$, $P(8914), P(38), P(914), P(39), P(314), P(89), P(814), P(38)(914), P(39)(814)$, $P(314)(89)$.

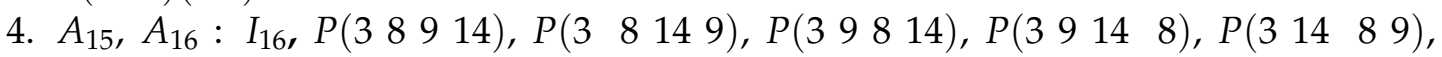
$P(3149$ 8), $P(398), P(314$ 8) $P(314$ 9), $P(38$ 14), $P(3149), P(3914), P(8149)$, $P(8914), P(38), P(914), P(39), P(314), P(89), P(814), P(38)(914), P(39)(814)$, $P(314)(89)$.

5. $A_{21}, A_{22}: I_{16}, P\left(\begin{array}{ll}4 & 13\end{array}\right), P\left(7\right.$ 10), $P\left(\begin{array}{lll}4 & 13\end{array}\right)\left(\begin{array}{ll}7 & 10\end{array}\right)$.

6. $A_{27}, A_{28}: I_{16}, P(3 \quad 9), P(814), P(3 \quad 9)(814)$.

Skew-symmetric matrix (secondary diagonal)

1. $A_{13}, A_{14}: P(25)$ (12 15), P (2 15) (5 12), P(2 512 15), P(2 1512 5).

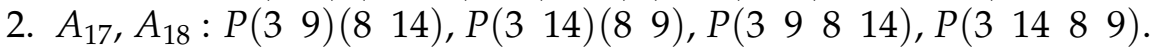

3. $A_{19}, A_{20}: P\left(\begin{array}{ll}3 & 8\end{array}\right)\left(\begin{array}{l}9 \\ 14\end{array}\right), P\left(\begin{array}{ll}3 & 9\end{array}\right)\left(\begin{array}{ll}8 & 14\end{array}\right), P\left(\begin{array}{llll}3 & 8 & 14 & 9\end{array}\right), P\left(\begin{array}{llll}3 & 9 & 14 & 8\end{array}\right)$.

4. $A_{23}, A_{24}: P\left(4\right.$ 10) (7 13), $P\left(\begin{array}{l}4 \\ 13\end{array}\right)$ (7 10), $P\left(\begin{array}{llll}4 & 10 & 7 & 13\end{array}\right), P\left(\begin{array}{llll}4 & 13 & 7 & 10\end{array}\right)$.

5. $A_{29}, A_{30}: P(4 \quad 7)\left(\begin{array}{ll}10 & 13\end{array}\right), P\left(\begin{array}{ll}4 & 13\end{array}\right)(7 \quad 10), P\left(\begin{array}{llll}4 & 10 & 13\end{array}\right), P\left(\begin{array}{lll}4 & 13 & 10\end{array}\right)$

6. $A_{5}, A_{6}: P(25)(1215), P(2$ 15) (5 12), P(l 512 15), P(2 1512 5).

\section{Conclusion}

It is found that there are 16 classifications of matrices in the Kronecker quaternion group. These classifications are divided into three types of matrices, namely

- A matrix having the same four entries has 24 commutation matrices.

- A matrix having the same two entries has four commutation matrices.

- symmetric skew matrix only has four commutation matrices 
Based on these results, a new study can be made by considering the elements in any matrix that have the same entries in certain desired positions.

\section{References}

[1] A. G. Kurosh, The Theory of Group, 2nd ed. American Mathematical Society, 2003.

[2] S. Rahayu, M. Soviana, Y. ., and A. Nazra, "Some Properties of Representation of Quaternion Group," KnE Engineering, vol. 1, no. 2, pp. 266-274, apr 2019, doi: http://dx.doi.org/10.18502/keg.v1i2.4451.

[3] Y. Yanita, M. R. Helmi, and A. M. Zakiya, "Solvability Group From Kronecker Product on the Representation of Quaternion Group," Asian Journal of Scientific Research, vol. 12, no. 2, pp. 293-297, mar 2019, doi: http:/ /dx.doi.org/10.3923/ajsr.2019.293.297.

[4] Yanita, "Other Properties and Presentation of Group from Kronecker Product on the Representation Quaternion Group," Journal of Computational and Theoretical Nanoscience, vol. 17, no. 2, pp. 874-877, feb 2020, doi: http://dx.doi.org/10.1166/jctn.2020.8734.

[5] A. Adrianda and Y. Yanita, "The first fundamental group of Kronecker quaternion group," Journal of Physics: Conference Series, vol. 1524, no. 1, p. 12039, apr 2020, doi: http://dx.doi.org/10.1088/1742-6596/1524/1/012039.

[6] A. M. Zakiya, Y. Yanita, and I. M. Arnawa, "Pictures on the second homotopy module of the group from Kronecker product on the representation quaternion group," Journal of Physics: Conference Series, vol. 1524, no. 1, p. 12038, apr 2020, doi: http://dx.doi.org/10.1088/1742-6596/1524/1/012038.

[7] K. Wang and P. Davis, "Group matrices for the quaternion and generalized dihedral groups," Computers and Mathematics with Applications, vol. 12, no. 5-6, pp. 1297-1301, sep 1986, doi: http://dx.doi.org/10.1016/0898-1221(86)90254-3.

[8] Ahsan, Y. Yanita, and S. Bahri, "Properties of the matrix from Kronecker product on the representation of quaternion group," Journal of Physics: Conference Series, vol. 1943, no. 1, p. 12125, jul 2021, doi: http://dx.doi.org/10.1088/1742-6596/1943/1/012125.

[9] D. A. Harville, Matrix Algebra from a statistician's Perpective. New York: Springer, 2008.

[10] J. A. Galian, Contemporary Abstract Algebra, 7th ed. Belmon, CA: Brooks/Cole, Cengage Learning, 2010.

[11] R. Piziak and P. L. Odell, Matrix Theory: From Generalized Inverses to Jordan Form. New York: Chapmann and Hall/CRC, 2007.

[12] C. $\mathrm{Xu}, \mathrm{L}$. He, and Z. Lin, "Commutation matrices and commutation tensors," Linear and Multilinear Algebra, vol. 68, no. 9, pp. 1721-1742, sep 2020, doi: http: //dx.doi.org/10.1080/03081087.2018.1556242.

[13] H. Zhang and F. Ding, "On the Kronecker Products and Their Applications," Journal of Applied Mathematics, vol. 2013, pp. 1-8, 2013, doi: http://dx.doi.org/10.1155/2013/296185.

[14] J. R. Magnus and H. Neudecker, "The Commutation Matrix: Some Properties and Applications," The Annals of Statistics, vol. 7, no. 2, pp. 381-394, 1979.

[15] - Matrix Diffrential Calculus with Applications in Statistics and Econometrics. New Jersey: John Wiley and Sons, Inc., 2007.

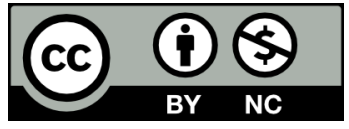

This article is an open-access article distributed under the terms and conditions of the Creative Commons Attribution-NonCommercial 4.0 International License. Editorial of JJoM: Department of Mathematics, Universitas Negeri Gorontalo, Jln. Prof. Dr. Ing. B.J. Habibie, Moutong, Tilongkabila, Kabupaten Bone Bolango, Provinsi Gorontalo 96119, Indonesia. 